\title{
An inactivated gE-deleted pseudorabies vaccine provides complete clinical protection and reduces virus shedding against challenge by a Chinese pseudorabies variant
}

Jichun Wang ${ }^{1,3}$, Rongli Guo ${ }^{1,3}$, Yongfeng Qiao ${ }^{1,3}$, Mengwei Xu ${ }^{1,3}$, Zhisheng Wang ${ }^{1,3}$, Yamei Liu ${ }^{1,3}$, Yiqi Gu ${ }^{1,2}$, Chang Liu, ${ }^{1,2}$ and Jibo Hou ${ }^{1,3^{*}}$

\begin{abstract}
Background: Since the end of 2011 an outbreak of pseudorabies affected Chinese pig herds that had been vaccinated with the commercial vaccine made of Bartha K61 strain. It is now clear that the outbreak was caused by an emergent PRV variant. Even though vaccines made of PRV Bartha K61 strain can confer certain cross protection against PRV variants based on experimental data, less than optimal clinical protection and virus shedding reduction were observed, making the control or eradication of this disease difficult.

Results: An infectious clone of PRV AH02LA strain was constructed to generate a gE deletion mutant PRV(LA-A $\left.{ }^{B}\right)$ strain. PRV $\left(L A-A^{B}\right)$ strain can reach a titer of $10^{8.43} \mathrm{TCID}_{50} / \mathrm{mL}$ (50\% tissue culture infectious dose) on BHK-21 cells. To evaluate the efficiency of the inactivated vaccine made of PRV $\left(L A-A^{B}\right)$ strain, thirty 3-week-old PRV-negative piglets were divided randomly into six groups for vaccination and challenge test. All five piglets in the challenge control showed typical clinical symptoms of pseudorabies post challenge. Sneezing and nasal discharge were observed in four and three piglets in groups C(vaccinated with inactivated PRV Bartha K61 strain vaccine) and $D$ (vaccinated with live PRV Bartha K61 strain vaccine) respectively. In contrast, piglets in both groups A(vaccinated with inactivated PRV LA-AB strain vaccine) and $B$ (vaccinated with inactivated PRV LA-A ${ }^{B}$ strain vaccine with adjuvant) presented mild or no clinical symptoms. Moreover, viral titers detected via nasal swabs were approximately 100 times lower in group B than in the challenge control, and the duration of virus shedding (3-4 days) was shorter than in either the challenge control (5-10 days) or groups $C$ and $D$ ( $5-6$ days).
\end{abstract}

Conclusions: The infectious clone constructed in this study harbors the whole genome of the PRV variant AH02LA strain. The gE deletion mutant PRV $\left(L A-A^{B}\right)$ strain generated from PRV AH02LA strain can reach a high titer on BHK-21 cells. An inactivated vaccine of PRV $L A-A^{B}$ provides clinical protection and significantly reduces virus shedding post challenge, especially if accompanied by the adjuvant CVC1302. While Inactivated or live vaccines made of PRV Barth K61 strain can provide only partial protection in this test.

Keywords: Pseudorabies virus emerging variant, gE deletion, Inactivated vaccine, Adjuvant, Bacterial artificial chromosome, Challenge protection

\footnotetext{
* Correspondence: houjibo@jaas.ac.cn

${ }^{1}$ National Research Center of Engineering and Technology for Veterinary

Biologicals/Institute of Veterinary Medicine, Jiangsu Academy of Agricultural

Sciences, Nanjing, Jiangsu 210014, China

${ }^{3}$ Jiangsu Co-innovation Center for Prevention and Control of Important

Animal Infectious Diseases and Zoonoses, Yangzhou, China

Full list of author information is available at the end of the article
} 


\section{Background}

Pseudorabies, caused by pseudorabies virus(PRV), has leaded to significant economic losses to the pig industry in many countries. Pseudorabies is a porcine infectious disease that can be effectively controlled using gene deletion marked vaccines and serum monitoring, enabling differentiation of vaccinated from infected animals [1-3]. However, in 2011 an outbreak of pseudorabies affected Chinese pig herds that had been vaccinated with the standard PRV Bartha K61 strain [4-9]. It is now clear that the outbreak was caused by an emergent PRV variant [5-7, 9-13]. As a support for this finding, the widely used PRV Bartha K61 strain was shown to be incapable of providing complete protection against this new PRV variant in several experimental studies [4-8]. Thus, a more efficacious vaccine is perceived as a necessary tool to help affected herds revert to PRV virus negative status.

To develop an effective vaccine, gene deletion mutants were generated from the new PRV strain and evaluated for protection efficacy. Although live vaccine candidates based on $\mathrm{gE}, \mathrm{gE} / \mathrm{gI}$, or $\mathrm{TK} / \mathrm{gE} / \mathrm{gI}$ gene deletion mutants are assumed to be highly protective, the safety issues of these preparations have to be demonstrated by lengthy testing [4, 14-18]. Alternatively, inactivated version of vaccine from the same mutants are promising to provide clinical protection as well based on relevant data about inactivated Bartha strain.

In this study, LA-A ${ }^{B}$, a gE deleted mutant of the emerging PRV strain AH02LA, was constructed using a bacterial artificial chromosome clone. A vaccine utilizing this mutant was evaluated with and without adjuvant in regard to its ability to provide clinical protection and reduce virus shedding after lethal challenge.

\section{Methods}

\section{Cells and viruses}

BHK-21 cells (CVCC:CL5, from China Veterinary Culture Collection Center) and chicken embryo cells (CECs) (SPF chicken embryo eggs were from Beijing Merial Vital Laboratory Animal Technology Co., Ltd) were prepared using standard methods. The PRV Bartha K61 strain was kindly provided by Professor Ping Jiang of Nanjing Agricultural University. Virus cultures and stocks were prepared using CECs or BHK-21 cells before at $-70{ }^{\circ} \mathrm{C}$. Virus titers were determined by $\mathrm{TCID}_{50}$ on BHK-21 cells following a standard protocol. Viral DNA extraction and transfection of plasmid, virus or bacterial artificial chromosome(BAC) DNAs were performed as described previously $[19,20]$.

\section{Plasmid and BAC manipulation}

The mini-F transfer vector pHA2-pUC19-H1-H2 was constructed as described [16]. Plasmid and BAC DNAs were isolated using commercial kits (Invitrogen PureLink ${ }^{\odot}$
Quick Plasmid Miniprep and Qiagen Large-Construct Kit) following the manufacturer's instructions. Next, restriction fragment length polymorphism(RFLP) analyses of PRV BACs was conducted using the restriction endonucleases BamH I, Kpn I, Pst I and Sph I and digestion conditions recommended by the supplier (Takara). Electrocompetent E.coli cells (DH10B) were obtained commercially (Invitrogen). E.coli GS1783 was made electrocompetent using published protocols [21-23]. Electroporation was performed to transform viral or BAC DNA following established methods [23-25].

\section{PCR and sequencing}

A pair of primers (Table 1) was designed from reference sequence (GenBank:NC_006151.1) to amplify sequences

Table 1 Primers for PCR or sequencing

\begin{tabular}{|c|c|}
\hline Primer & Sequence \\
\hline PRV gD F & 5'-AACACCTAATTTGCGTACGGC-3' \\
\hline PRV gD R & 5'-TCATCATCGACGCCGGTACT-3' \\
\hline PRV gl F & 5'-TGGGCGTGTGCGTCTACATCT-3' \\
\hline PRV gl R & 5'-CAACCCCGGTGTGTGTGAGA-3' \\
\hline$\Delta \mathrm{gE} T^{\vee}$ overlap1 $\mathrm{F}$ & 5'-CCACGCCCAGCGGTCCATAAAATTGGGT-3' \\
\hline$\Delta g E T^{\vee}$ overlap1 R & $\begin{array}{l}5^{\prime}-\text { TCATCACGAAGGAGCCCAGCAAAGGG } \\
\text { CCGCATGG TCTCAACC-3' }\end{array}$ \\
\hline$\Delta \mathrm{gE} T^{\vee}$ overlap2 F & 5'-GCTGGGCTCCTTCGTGATGA-3' \\
\hline$\Delta g E T^{\vee}$ overlap2 $R$ & 5'-TCACGATCTGGGCATGCAGG-3' \\
\hline SEQ-gl/gE F 1 & 5'-TTGCGTACGGCCTTGCTTAC-3' \\
\hline SEQ-gl/gE F 2 & 5'-GACTACATGTTCCCCACGGA-3' \\
\hline SEQ-gl/gE F 3 & 5'-ACGCCGTACGCCATCGACCC-3' \\
\hline SEQ-gl/gE F 4 & 5'-ACGCTGCTGTTTCTGGAGGG-3' \\
\hline SEQ-gl/gE F 5 & 5'-ACGAAGAGGAGGAGGACGAG-3' \\
\hline SEQ-gl/gE F 6 & 5'-ACCATGCGGCCCTTTCTGCT-3' \\
\hline SEQ-gl/gE F 7 & 5'-ACCACGGTGTGCTTCGAGAC-3' \\
\hline SEQ-gl/gE F 8 & 5'-TGTACGAGCCCTGCATCTACCACCC-3' \\
\hline SEQ-gl/gE F 9 & 5'-ACTACTACGACGGCGACGACGACGA-3' \\
\hline SEQ-gl/gE F 10 & 5'-AACGAGACGCCCAGCGAGTT-3' \\
\hline SEQ-gl/gE F 11 & 5'-AAGGTGCTCACCGAGTGGTGCTACG-3' \\
\hline SEQ-gl/gE R 1 & 5'-TCTAGGAGATGGTACATCGCGGGGC-3' \\
\hline SEQ-gl/gE R 2 & 5'-TGGTGATGTAGAACGGCGCC-3' \\
\hline SEQ-gl/gE R 3 & 5'-ACAGCGAGCAGATGACCAGC-3' \\
\hline SEQ-gl/gE R 4 & 5'-TCGCTGCTGAACTCGTCCTC-3' \\
\hline SEQ-gl/gE R 5 & 5'-ATCACGAGCACGTACAGCCC-3' \\
\hline SEQ-gl/gE R 6 & 5'-TGTAGAGGCCCGTGTCGTTG-3' \\
\hline SEQ-gl/gE R 7 & 5'-AAAGGGCCGCATGGTCTCAACC-3' \\
\hline SEQ-gl/gE R 8 & 5'-TCCTCCTCCTCTTCGTCGGA-3' \\
\hline SEQ-gl/gE R 9 & 5'-AAAGAGGTCCGTGGTCCCGTTCAC-3' \\
\hline SEQ-gl/gE R 10 & 5'-AGATGTAGACGCACACGCCCACCAG-3' \\
\hline SEQ-gl/gE R 11 & 5'-GGGAACATGTAGTCCGCGGA-3' \\
\hline
\end{tabular}


of gI gene with isolated PRV AH02LA strain DNA as template. These primers were also used for sequencing gI gene (GeneScript, Nanjing China). To obtain the gE deletion, overlap PCR was conducted with two pairs of primers, $\Delta \mathrm{gE} \mathrm{T} \mathrm{T}^{\mathrm{V}}$ overlap1 and $\Delta \mathrm{gE} \mathrm{T} \mathrm{T}^{\mathrm{V}}$ overlap2 (Table 1). Briefly, with PRV AH02LA DNA as template, two PCR reactions were performed separately with the above two primer pairs. Next, the PCR products were gel purified to serve as templates in another PCR reaction with primers $\triangle g E \mathrm{~T}^{\mathrm{V}}$ overlap1 $\mathrm{F}$ and $\Delta \mathrm{gE} \mathrm{T} \mathrm{T}^{\mathrm{V}}$ overlap2 $\mathrm{R}$ to produce a fragment for $\mathrm{gE}$ deletion mutant generation. Thus the generated mutant, consists of upstream sequences, the intact gI gene, part of gE gene(1299 bp to 1735 bp of gE ORF), and downstream sequences (Fig. 1). Finally, the primers PRV Homo-1 F and PRV Homo-2R [16] were used to amplify the repairing viral DNA with the PRV AH02LA DNA as template.

\section{Multistep growth kinetics}

Multistep growth kinetics of parental AH02LA, gE deletion LA-A ${ }^{\mathrm{B}}$, and repair AH02LA ${ }^{\mathrm{R}}$ viruses were tested on BHK-21 cells following methods as described [26] on monolayers $\left(1 \times 10^{6}\right.$ cells $)$ of BHK-21 cells with a multiplicity of infection(MOI)of 0.01 . Three independent experiments were conducted and one-way ANOVA (SPSS software package) was employed to identify significant differences.

\section{$\mathrm{LD}_{50}$ determination for PRV AH02LA strain}

To determine the $\mathrm{LD}_{50}$ of the virulent PRV AH02LA strain, the virus $\left(\operatorname{TCID}_{50}=10^{-8.25} / \mathrm{mL}\right)$ was serially diluted with DMEM to $10^{-1}, 10^{-2}, 10^{-3}$ and $10^{-4}$. Twentyfive 77-day-old piglets were randomly divided into five groups. Piglets in each challenge group were inoculated intranasally(I.N.) with $1 \mathrm{~mL}$ diluted virus of different titers. Animals were observed for additional fourteen days. The number of piglets that died in each group was recorded to calculate $\mathrm{LD}_{50}$ using the Karber method.

\section{Construction of PRV BAC and deletion mutants}

The PRV BAC was constructed from an isolate of the emergent PRV variant following published methods (Fig. 1) $[16,26]$. Briefly, After cotransfection of $\sim 1.5 \mu \mathrm{g}$ PRV AH02LA strain DNA and $4 \mu \mathrm{g}$ pHA2-pUC19-H1-H2 DNA, mini-F recombinant viruses with green fluorescence were purified to obtain a homogeneous population [27]. Next, mini-F containing PRV DNA was isolated and then transferred into E. coli cells DH10B and subsequently into E.coli strain GS1783 cells by electroporation for mutagenesis [23]. Finally, BAC DNA was isolated and RFLPs were identified as described [24] using BamH I, Kpn I, Pst I and

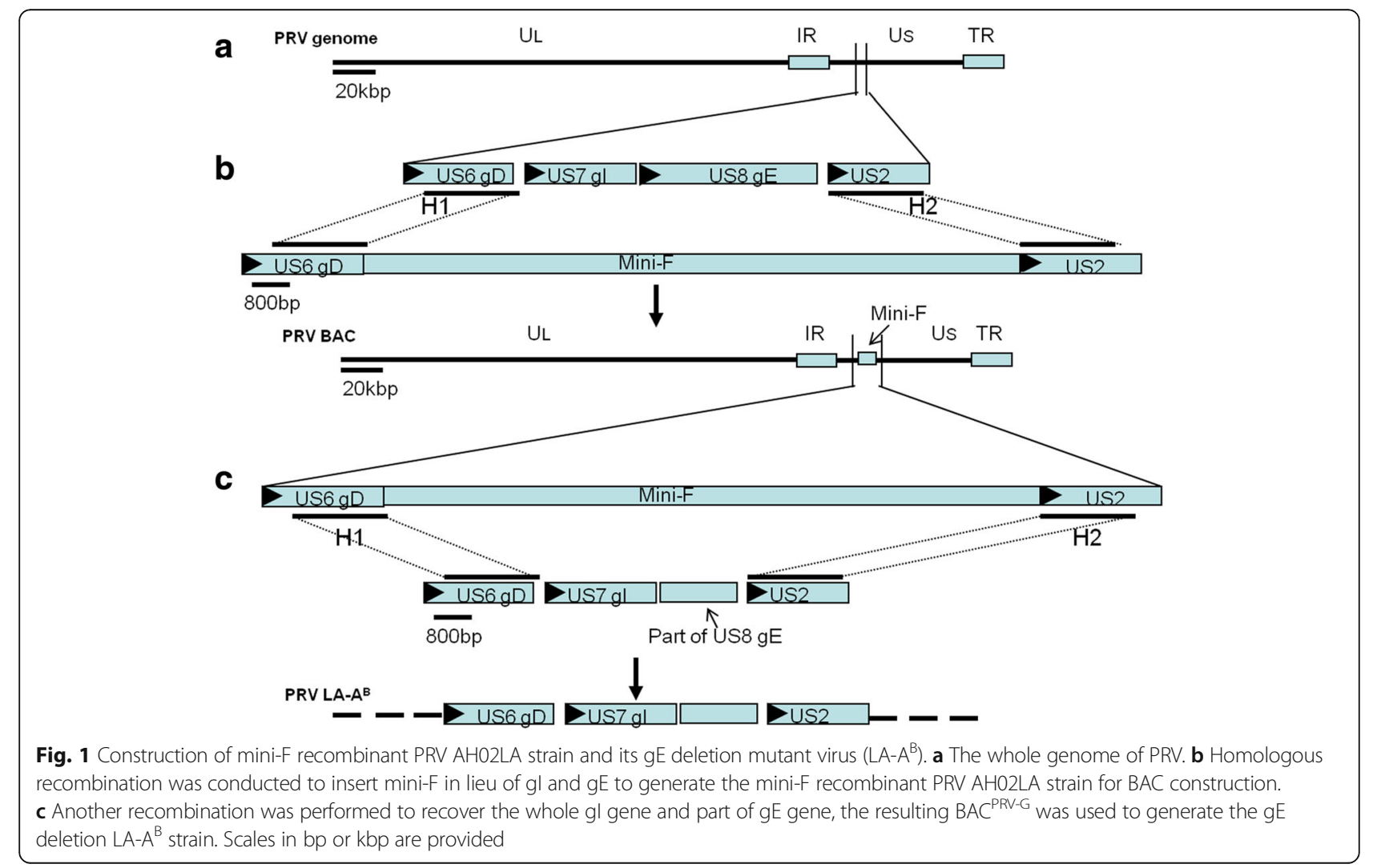


Sph I, using the complete genome sequence of PRV ZJ01 strain (GenBank: KM061380.1) as a reference for in silico prediction.

To generate the gE deletion mutant of PRV AH02LA strain, another homologous recombination was performed to recover the gI gene and the undeleted sequences of $\mathrm{gE}$ as shown in Fig. 1. Briefly, PCR with primers $\triangle \mathrm{gE} \mathrm{T} \mathrm{T}^{\mathrm{V}}$ overlap1 $\mathrm{F}$ and $\triangle \mathrm{gE} \mathrm{T}^{\mathrm{V}}$ overlap2 $\mathrm{R}$ (Table 1) was performed with the PRV AH02LA DNA as template. And then CECs were co-transfected with approximately $5 \mu \mathrm{g}$ PRV BAC DNA and $2 \mu \mathrm{g}$ DNA of fragment from the second PCR reaction of overlap PCR with primers $\Delta \mathrm{gE} \mathrm{T}^{\mathrm{V}}$ overlap1 $\mathrm{F}$ and $\Delta \mathrm{gE} \mathrm{T}^{\mathrm{V}}$ overlap2 $\mathrm{R}$ by calcium phosphate precipitation. Using illumination at $488 \mathrm{~nm}$, non-fluorescent virus isolates were selected and purified to homogeneity. Product structure was confirmed by sequencing with the same primers and other specific primers from the SEQ-gI/gE series (Table 1).

For generation of the gI/gE repair virus via homologous recombination, CECs were transfected with approximately $5 \mu \mathrm{g}$ PRV BAC DNA and $2 \mu \mathrm{g}$ PCR product generated using DNA of parental PRV as template and primers matching PRV Homo $1 \mathrm{~F}$ and PRV Homo 2R [16] (Fig. 1). Two to three days after transfection, nonfluorescent plaques $(488 \mathrm{~nm})$ were isolated and purified to obtain a homogeneous population. The structure of the recovered viruses was confirmed by PCR and sequencing with primers PRV Homo $1 \mathrm{~F}$, PRV Homo 2R and sequencing primers of SEQ-gI/gE (Table 1).

\section{Preparation of vaccine}

PRV LA- $A^{B}$ and Bartha K61 viruses were propagated in BHK-21 cells using a 5 liter bioreactor, each virus stock was diluted to $10^{8.5} \mathrm{TCID}_{50} / \mathrm{mL}$. After inactivation with formalin (Sigma-Aldrich) as described [16], vaccines were prepared using mineral oil adjuvant (1:3 water in oil). All vaccines were confirmed to be free of bacteria and fungi, and had the expected physical properties according to standard protocols. Vaccines were stored at $4{ }^{\circ} \mathrm{C}$ until use.

\section{Test of vaccine efficacy}

Thirty piglets of 21-day-old (from Zhengzhuquan Pig Breeding Farm in Pukou district, Nanjing, China) were randomly divided into 6 groups (A-F) and housed in separated facilities. All piglets were antibody negative for $\mathrm{PRV} g B$ and $\mathrm{gE}$, and free of porcine reproductive and respiratory syndrome virus, porcine parvovirus, porcine circovirus 2 and classical swine fever virus. All inoculations were given intramuscularly(I.M.) at $2 \mathrm{~mL} / \mathrm{pig}$. Group A piglets were inoculated with LA-A ${ }^{\mathrm{B}}$, group B piglets with LA-A ${ }^{B}$ plus adjuvant CVC1302 (Patent: CN103083663B, kindly provided by the group of Dr. Qisheng Zheng in our institute), group $C$ piglets with inactivated Bartha K61, group D piglets with live Bartha
K61 of $1 \times 10^{5.0} \mathrm{TCID}_{50}$ virus/dose, and groups $\mathrm{E}$ and $\mathrm{F}$ piglets were dosed with PBS only. Piglets in groups A, B, and $\mathrm{C}$ were inoculated at 28 days of age and again at 56 days. Group D piglets were inoculated once, at 28-dayold. Group E and F piglets were dosed once, at 28 days of age. Three weeks after the second inoculation (at which time the piglets were 11-week-old), groups $\mathrm{A}, \mathrm{B}, \mathrm{C}, \mathrm{D}$ and E were challenged I.N. with $3 \times \mathrm{LD}_{50}$ PRV AH02LA per piglet. Group F piglets were not challenged, serving as a negative control. Serum samples from all piglets were collected before inoculation, and at 7, 14, 21, 28, 35, 42 and 49 days post inoculation, and again at 14 days post challenge(P.C.). Serum was subjected to ELISA tests for PRV gB and $\mathrm{gE}$ antibodies, and cross-neutralizing (NA) antibodies against AH02LA or Bartha K61. Body temperature, clinical signs, and virus shedding were monitored and recorded daily until 14 days P.C.. The presence of lung lesions for died or survived piglets was noted at the end of the test.

\section{Test for serological antibodies}

ELISA tests were performed with the PRV gE or gB antibody detection kit (IDEXX, Maine, USA) following manufacturer's instructions. Cross neutralization tests were conducted according to the standard methods published by OIE (Veterinary Pharmacopoeia of the People's Republic of China (2010)) with slight modification. Briefly, serum samples were diluted two-fold with PBS, mixed with 100 TCID $_{50}$ PRV AH02LA or PRV Bartha K61 virus, and incubated for $1 \mathrm{~h}$ at $37{ }^{\circ} \mathrm{C}$. The serum-virus mixture was used to inoculate BHK-21 cells which were then incubated at $37{ }^{\circ} \mathrm{C}, 5 \% \mathrm{CO}_{2}$ for 3-4 days to observe the development of CPE. Titers of neutralizing antibody were expressed as $\log _{2}$ of highest dilution at which no $\mathrm{CPE}$ was observed, and mean titers of each group were calculated for comparison.

\section{Detection of virus shedding}

Nasal swab samples were collected from all piglets before challenge and daily to 14 days after challenge. After shaking at 5000-8000 RPM for 1-2 min, one freezethaw cycle $\left(-70{ }^{\circ} \mathrm{C}\right.$ and $\left.37{ }^{\circ} \mathrm{C}\right)$ was conducted to release viruses from nasal swabs. Samples were centrifuged at 10,000 RPM for $15 \mathrm{~min}$ to pellet tissue and cell debris, the supernatants were collected to determine virus titers. Viruses titers were expressed as $\mathrm{TCID}_{50}$ following the method of Karber.

All animal studies were conducted following guidelines provided by the Institutional Biosafety Committee and approved by the Institutional Animal Care and Use Committee at the Jiangsu Academy of Agriculture Sciences. Experiments involving virulent PRV were conducted under Biosafety Level 2+ containment. 


\section{Results}

Isolation and identification of an emergent pseudorabies virus variant (AH02LA strain)

BHK-21 cells, inoculated with a sample of brain tissue from a stillborn piglet, exhibited cytopathogenic effects (CPE) after 3 days. After three rounds of plaque purification, a homogeneous population of virus was isolated and designated as the AH02LA strain. Using the viral DNA as template and the primers PRV gI F/ R (Table 1), PCR generated a product of 1250-1400 bp. Sequencing established that this fragment was homologous to the PRV gI gene. The AH02LA strain can be specifically neutralized with the standard antibodies against PRV (CIVDC, Beijing China) with a neutralizing index of 10,000. These results demonstrate that a variant PRV field isolate was identified.

\section{Pathology of the PRV AH02LA strain}

The $\mathrm{LD}_{50}$ of PRV AH02LA (Batch: JJ-R5) was determined using 77-day-old piglets free of antibodies against PRV. Three of five piglets in the $10^{-5}$ dosage group, and all of the piglets in the $10^{-4}$ dosage group, showed typical symptoms of PRV infection: sneezing, purulent nasal discharge, difficulty in breathing and ataxia, indicating the high virulence of this strain. The $\mathrm{LD}_{50}$ was $10^{-2.32}$ / $\mathrm{mL}$. The highly virulent character of AH02LA is shared by other emergent PRV strains isolated during the 2011 outbreaks [4-8]. The significant difference between these emerging strains and the conventional strain $\mathrm{S}$
(HWBD, Harbin China) is that the former cause mortalities in not only young piglets but also much older pigs, whereas the conventional strain $\mathrm{S}$ causes death only in young (less than 15-day-old) piglets (Veterinary Pharmacopoeia of the People's Republic of China (2005)).

\section{Construction of a bacterial artificial chromosome containing the AH02LA genome}

Green plaques of mini-F recombinant PRV AH02LA were observed under UV light $(488 \mathrm{~nm})$, and isolated after several rounds of plaque purification. The mini-F recombinant PRV AH02LA DNA was isolated and transfected into Escherichia coli DH10B competent cells, the resulting clone was designated $\mathrm{BAC}^{\mathrm{PRV}-\mathrm{AH} 02 \mathrm{LA}}$. BAC $\mathrm{BRV}^{\mathrm{PRV}}$ AH02LA DNA was electroporated into E. coli GS1783 competent cells, resulting in a clone designated $B A C^{\text {PRV-G }}$. This BAC was used for further gene manipulations using the En Passant protocol [20]. Restriction fragment length polymorphism (RFLP) analysis of BAC ${ }^{\text {PRV-G }}$ with $\mathrm{BamH}$ I, Kpn I, Pst I and Sph I generated patterns similar to those predicted from the PRV ZJ01 strain sequence (GenBank: KM061380.1) with minor differences (Fig. 2). Virus was rescued successfully by transfection of the $B A C^{\text {PRV-G }}$ DNA into primary CECs, designated PRV AH02LA ${ }^{R}$. In summary, an infectious clone containing the complete genome of the PRV emerging variant AH02LA strain was constructed successfully.

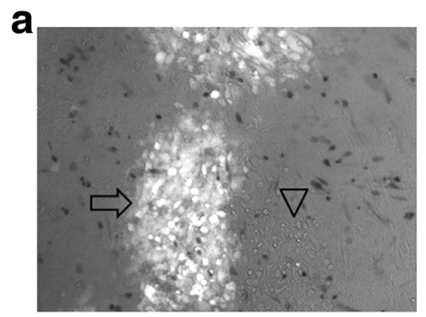

Fluorescence under UV excitation

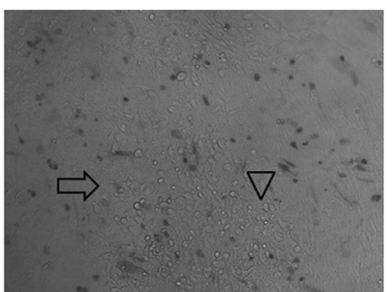

Phase contrast
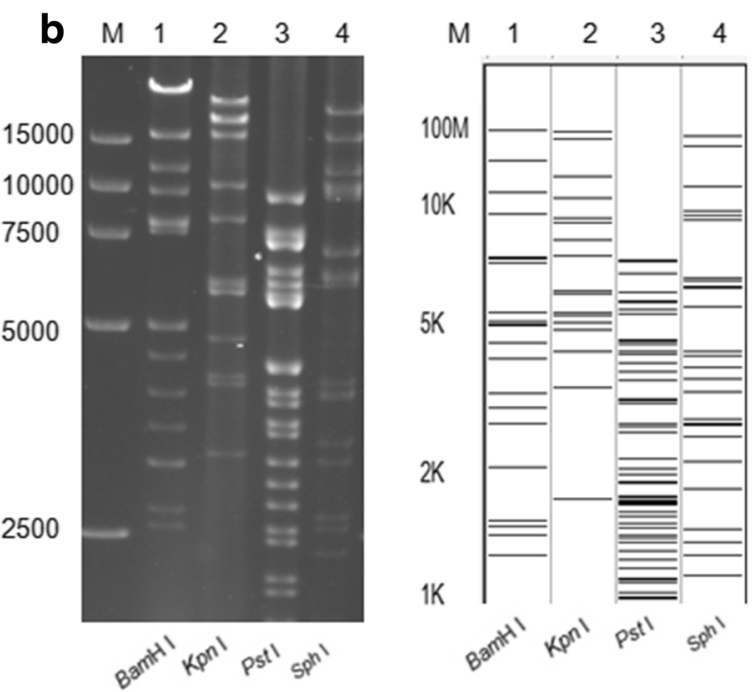

Fig. 2 Plaques of mini-F recombinant PRV AH02LA strain and RFLP of BAC ${ }^{P R V-G}$. a Images of mini-F recombinant PRV AH02LA and the parental AH02LA plaques under UV excitation (upper) and phase contrast (lower) are shown. Arrowhead shows a plaque of parental PRV AH02LA virus and arrow shows a plaque formed by mini-F recombinant PRV AH02LA. Each panel represents a view of $200 \times 200 \mu \mathrm{m}$ in size. $\mathbf{b}$ RFLP of BACPRV-G DNA from PRV AH02LA BAC clone BAC ${ }^{\text {PRV-G }}$ was prepared by mini-prep and digested with BamH I, Kpn I, Pst I and Sph I (lanes 1-4). The digests were separated by $0.8 \%$ agarose gel electrophoresis for $16 \mathrm{~h}$ under $40 \mathrm{~V}$ (Left). Predicted RFLP patterns of BACPRV-G with BamH I, Kpn I, Pst I and Sph I digestion respectively. Predictions of these digestions were performed with the whole genome sequence of PRV ZJ01 strain (GenBank: KM061380.1) as reference. M: DL 15,000 DNA Marker (Takara) 


\section{Construction of a gE deletion mutant virus}

After co-transfection of BAC ${ }^{\mathrm{PRV}-\mathrm{G}}$ DNA with a DNA fragment containing the $\mathrm{gE}$ deletion (obtained through PCR), several white plaques were observed under UV illumination at $488 \mathrm{~nm}$. One was isolated after several rounds of plaque purification and designated PRV LA$A^{B}$. The deletion was verified by PCR using primers of $\triangle \mathrm{gE} T \mathrm{TV}$ overlap1 $\mathrm{F}$ and $\Delta \mathrm{gE} T \mathrm{TV}$ overlap2 $\mathrm{R}$, followed by sequencing with the appropriate primers (Table 1).

\section{Growth kinetics of the PRV LA-A $A^{B}$ virus}

Multistep growth kinetics for the PRV LA-A ${ }^{\mathrm{B}}$, AH02LA, and AH02LA ${ }^{R}$ viruses were determined. Peak supernatant titers for LA-A ${ }^{\mathrm{B}}$, AH02LA, and AH02LA ${ }^{\mathrm{R}}$ were $10^{8.18}, 10^{9.01}$, and $10^{8.19} \mathrm{TCID}_{50} / \mathrm{mL}$ respectively. At 48 hpi the titers for LA-A ${ }^{B}$ and AH02LA ${ }^{R}$ were not significantly different from AH02LA $(p=0.414$ and 0.362). For cell-associated virus titers $\mathrm{LA}^{\mathrm{B}} \mathrm{B}^{\mathrm{B}}, \mathrm{AH} 02 \mathrm{LA}$, and AH02LA ${ }^{\mathrm{R}}$ were $10^{8.43}, 10^{8.86}$, and $10^{8.13} \mathrm{TCID}_{50} / \mathrm{mL}$ at $48 \mathrm{hpi}$, respectively. The titers of LA- ${ }^{\mathrm{B}}$ and AH02LA ${ }^{\mathrm{R}}$ are not significant different from the parental virus AH02LA at 48 hpi ( $p=0.126$ and 0.088$)$.

\section{Immunogenicity of the LA-A ${ }^{B}$ vaccine}

In challenge control group, all piglets had typical clinical syndromes of pseudorabies infection including sneezing, coughing, difficulty breathing and nasal discharge P.C.. Two piglets died at 7 days and two at 8 days P.C. (Table 2). Body temperatures of these piglets reached over $41^{\circ} \mathrm{C}$ and lasted for 4-5 days (Fig. 3(a)). All the piglets shed virus with titers of $10^{0.25} \sim 10^{5.25} \mathrm{TCID}_{50} / 0.1 \mathrm{~mL}$ lasting for 5 7 days till death or 10 days for the surviving piglet (Fig. 3(b)). All piglets showed severe lung lesions from hemorrhaging and congestion (Table 2).

$\mathrm{gB}$ antibodies were detected in all piglets vaccinated with live Bartha $\mathrm{K} 61$ strain or the LA-A ${ }^{\mathrm{B}}$ strain plus adjuvant at 7 days post vaccination(P.V.), while two of five piglets vaccinated $\mathrm{LA}-\mathrm{A}^{\mathrm{B}}$ strain alone or the inactivated Bartha K61 strain were anti-gB positive at 7 days P.V.. All samples collected at 14 days P.V. were positive for $\mathrm{gB}$ antibodies (Table 2). This result indicates the adjuvant CVC1302 helps the LA-A ${ }^{B}$ vaccine stimulate immunity against PRV more quickly. At 14 days P.C. all piglets were positive for $\mathrm{gE}$ antibodies (Table 2).

No neutralizing antibodies (NA) against the AH02LA or Bartha K61 strain, were detected in any of the piglets at 7 days P.V.. At 14 days P.V. neutralizing antibodies were detected in one piglet from group $B\left(L A-A^{B}\right.$ plus adjuvant) at a titer of 2 . In groups $A, B$, and $C, N A$ titers began increasing quickly seven days after the boost and continued to increase over the next two weeks. The LA- $\mathrm{A}^{\mathrm{B}}$ vaccines induced higher titers of NA against the AH02LA and Bartha K61 strain, than vaccines made of Bartha K61.
One dose of live Bartha K61 vaccine stimulated a weak NA titer of less than 4 against the AH02LA strain or the Bartha K61 strain. These results indicate that optimizing the prime-boost regimen is necessary for live Bartha K61 vaccine to stimulate strong humoral immunity. NA titers in group B piglets were higher than other groups, indicating that adjuvant $\mathrm{CVC1302}$ helped stimulate higher specific immunity for LA- $\mathrm{A}^{\mathrm{B}}$ vaccine (Table 2 ).

All piglets in the vaccinated groups (A, B, C and D) were protected against lethal challenge. Fevers in group $A$ and $B$ piglets were transient, and other clinical symptoms were mild or absent respectively. Titers of shed virus in $\mathrm{LA}-\mathrm{A}^{\mathrm{B}}$ vaccinated group A piglets were $10^{0.25} \sim$ $10^{3.75} \mathrm{TCID}_{50} / 0.1 \mathrm{~mL}$ lasting $3-4$ days; in $\mathrm{LA}-\mathrm{A}^{\mathrm{B}}$ plus adjuvant group $\mathrm{B}$ piglets, titers were $10^{0.125} \sim 10^{2.875}$ $\mathrm{TCID}_{50} / 0.1 \mathrm{~mL}$ lasting 3-4 days. Piglets in groups $\mathrm{C}$ and D (vaccinated with inactivated Bartha $\mathrm{K} 61$ and live Bartha K61 respectively) presented fevers of more than $41{ }^{\circ} \mathrm{C}$ lasting for 4-6 days (Fig. 3(a)), four and three piglets respectively, presented sneezing lasting for 2-4 days and nasal discharge lasting 2-3 days (Table 2). The titers of shed virus in these piglets were $10^{0.125} \sim 10^{4.125}$ $\mathrm{TCID}_{50} / 0.1 \mathrm{~mL}$ lasting for 5-6 days (Fig. 3(b)). No Lung lesions were observed in any group A or B piglets, while $2 / 5$ group $C$ piglets and in $1 / 5$ group $D$ piglets presented lesions from hemorrhage and congestion (Table 2).

\section{Discussion}

The results of vaccination test demonstrate that vaccines made of PRV LA- $A^{B}$ elicit a more effective immune response against the emergent PRV AH02LA than the Bartha K61 vaccines. The immune response is even more pronounced when PRV LA- $A^{B}$ is administered with adjuvant. Pigs vaccinated with $\mathrm{LA}-\mathrm{A}^{\mathrm{B}}$ produced lower titers of virus shedding over a shorter duration than pigs with Bartha K61. This is a clear advantage in the terms of controlling the spread of infection. Adjuvant CVC1302 is a mixture of monophosphoryl lipid A (MPLA), muramyl dipeptide (MDP) and $\beta$-glucan. This mixture has been shown to improve the efficiency of the inactivated vaccines of porcine epidemic diarrhea virus and foot and mouth disease virus (Patent:CN103083663B). MPLA, MDP and $\beta$-glucan can serve as delivery systems or stimulators for the immune system [28-31].

Bacterial artificial chromosome(BAC) of herpesvirus is a useful tool for generation of gene modified mutants to study the mechanism of the viruses. After the construction of the first BAC of mouse cytomegalovirus (MCMV) [32], a few of herpesvirus genomes have been maintained in BACs as infectious clones, which have provided great help for discovery of pathology of these viruses [27, 33] and construction of vectored vaccines $[26,34,35]$, especially following the protocol of En Passant protocol [23]. The parental PRV AH02LA strain of 
Table 2 Antibodies, clinical signs and lung lesions in animals

\begin{tabular}{|c|c|c|c|c|c|c|c|c|}
\hline \multicolumn{3}{|l|}{ Groups } & \multirow{2}{*}{$\frac{\mathrm{A}}{0^{\mathrm{a}} / 5^{\mathrm{b}}}$} & \multirow{2}{*}{$\frac{B}{0 / 5}$} & \multirow{2}{*}{$\frac{C}{0 / 5}$} & \multirow{2}{*}{$\frac{D}{0 / 5}$} & \multirow{2}{*}{$\frac{E}{0 / 5}$} & \multirow{2}{*}{$\frac{F}{0 / 5}$} \\
\hline ELISA antibodies against & B.V. & $g B+$ & & & & & & \\
\hline & & $\mathrm{gE}+$ & $0 / 5$ & $0 / 5$ & $0 / 5$ & $0 / 5$ & $0 / 5$ & $0 / 5$ \\
\hline & 7d P.V. & $\mathrm{gB}+$ & $2 / 5$ & $5 / 5$ & $2 / 5$ & $5 / 5$ & $0 / 5$ & $0 / 5$ \\
\hline & & $\mathrm{gE}+$ & $0 / 5$ & $0 / 5$ & $0 / 5$ & $0 / 5$ & $0 / 5$ & $0 / 5$ \\
\hline & 14d P.V. & $g B+$ & $5 / 5$ & $5 / 5$ & $5 / 5$ & $5 / 5$ & $0 / 5$ & $0 / 5$ \\
\hline & & $\mathrm{gE}+$ & $0 / 5$ & $0 / 5$ & $0 / 5$ & $0 / 5$ & $0 / 5$ & $0 / 5$ \\
\hline & 21d P.V. & $\mathrm{gB}+$ & $5 / 5$ & $5 / 5$ & $5 / 5$ & $5 / 5$ & $0 / 5$ & $0 / 5$ \\
\hline & & $\mathrm{gE}+$ & $0 / 5$ & $0 / 5$ & $0 / 5$ & $0 / 5$ & $0 / 5$ & $0 / 5$ \\
\hline & 14d P.C. & $\mathrm{gB}+$ & $5 / 5$ & $5 / 5$ & $5 / 5$ & $5 / 5$ & $2 / 2$ & $0 / 5$ \\
\hline & & $\mathrm{gE}+$ & $5 / 5$ & $5 / 5$ & $5 / 5$ & $5 / 5$ & $2 / 2$ & $0 / 5$ \\
\hline \multirow{16}{*}{$\begin{array}{l}\text { Mean titers of } \\
\text { neutralizing antibodies }\end{array}$} & AH02LA & B.V. & $<2^{c}$ & $<2$ & $<2$ & $<2$ & $<2$ & $<2$ \\
\hline & & 7d P.V. & $<2$ & $<2$ & $<2$ & $<2$ & $<2$ & $<2$ \\
\hline & & 14d P.V. & $<2$ & 0.4 & $<2$ & $<2$ & $<2$ & $<2$ \\
\hline & & 21d P.V. & 2.00 & 2.80 & $<2$ & 1.20 & $<2$ & $<2$ \\
\hline & & 28d P.V. & 2.60 & 3.40 & 2.40 & 2.60 & $<2$ & $<2$ \\
\hline & & 35d P.V. & 4.60 & 5.20 & 3.60 & 3.20 & $<2$ & $<2$ \\
\hline & & 42d P.V. & 4.60 & 5.60 & 3.80 & 3.20 & $<2$ & $<2$ \\
\hline & & 49d P.V. & 5.20 & 6.20 & 4.20 & 3.00 & $<2$ & $<2$ \\
\hline & Bartha K61 & B.V. & $<2$ & $<2$ & $<2$ & $<2$ & $<2$ & $<2$ \\
\hline & & 7d P.V. & $<2$ & $<2$ & $<2$ & $<2$ & $<2$ & $<2$ \\
\hline & & 14d P.V. & $<2$ & 0.4 & $<2$ & $<2$ & $<2$ & $<2$ \\
\hline & & 21d P.V. & 1.20 & 2.24 & 1.2 & 1.60 & $<2$ & $<2$ \\
\hline & & 28d P.V. & 2.40 & 3.08 & 2.60 & 3.20 & $<2$ & $<2$ \\
\hline & & 35d P.V. & 4.60 & 4.80 & 4.60 & 3.20 & $<2$ & $<2$ \\
\hline & & 42d P.V. & 4.80 & 5.20 & 4.80 & 3.60 & $<2$ & $<2$ \\
\hline & & 49d P.V. & 5.40 & 5.60 & 5.20 & 3.40 & $<2$ & $<2$ \\
\hline \multirow{4}{*}{$\begin{array}{l}\text { Clinical signs and lung } \\
\text { lesions post challenge }\end{array}$} & \multicolumn{2}{|l|}{ Morbidity } & $1 / 5$ & $0 / 5$ & $4 / 5$ & $3 / 5$ & $5 / 5$ & $0 / 5$ \\
\hline & \multicolumn{2}{|c|}{ Duration of clinical signs(days) } & 1 & - & $2-4$ & $2-3$ & $4-7$ & - \\
\hline & \multicolumn{2}{|l|}{ Mortality } & $0 / 5$ & $0 / 5$ & $0 / 5$ & $0 / 5$ & $4 / 5$ & $0 / 5$ \\
\hline & \multicolumn{2}{|c|}{ Lung lesions } & $0 / 5$ & $0 / 5$ & $2 / 5$ & $1 / 5$ & $5 / 5$ & $0 / 5$ \\
\hline
\end{tabular}

B.V means before vaccination, P.V means post vaccination, P.C means post challenge. "a" indicates the number of piglets positive; $b$ " indicates the number of piglets in the group. " $\mathrm{C}$ " indicates mean titers of neutralizing antibody of each group expressed as nlog ${ }_{2}$ of highest dilution at which no CPE was observed

the BAC constructed in this study is a virulent variant strain that caused severe losses in many porcine farms in China. So even though En Passant protocol was not performed in the generation of the gE deleted LA- $\mathrm{A}^{\mathrm{B}}$ strain in this study, it will be used for further analysis of the mechanism of the high virulence of this variant.

The latent infection of PRV is always a potential threat to porcine herds [36]. So the eradication of PRV has been a direct aim of many farms for control of this disease. This aim has been achieved in a few countries [1] and the infection of wild PRV has been controlled to very low levels in some individual farms in China before 2011 [37, 38]. DIVA vaccine(vaccine allowing differentiating infected from vaccinated animals) has played an important role towards this aim [1]. In the project of eradication of PRV, the efficient reduction of virus shedding post infection with virulent strain is crucial [2]. In this study, the inactivated vaccine of LA- ${ }^{B}$ strain with adjuvant $\mathrm{CVC1302}$ reduced virus shedding significantly, despite that all vaccinated animals did not stop virus shedding post challenge.

We did find a difference in the profile of clinical symptoms between animals challenged through I.M. or I.N. route. During the challenge test in this study, we also tried the challenge route of I.M. injection for 11-weekold piglets (data not shown). We found that even though it caused high fever and obvious clinical syndromes of PR through this way, the virulence was significantly 


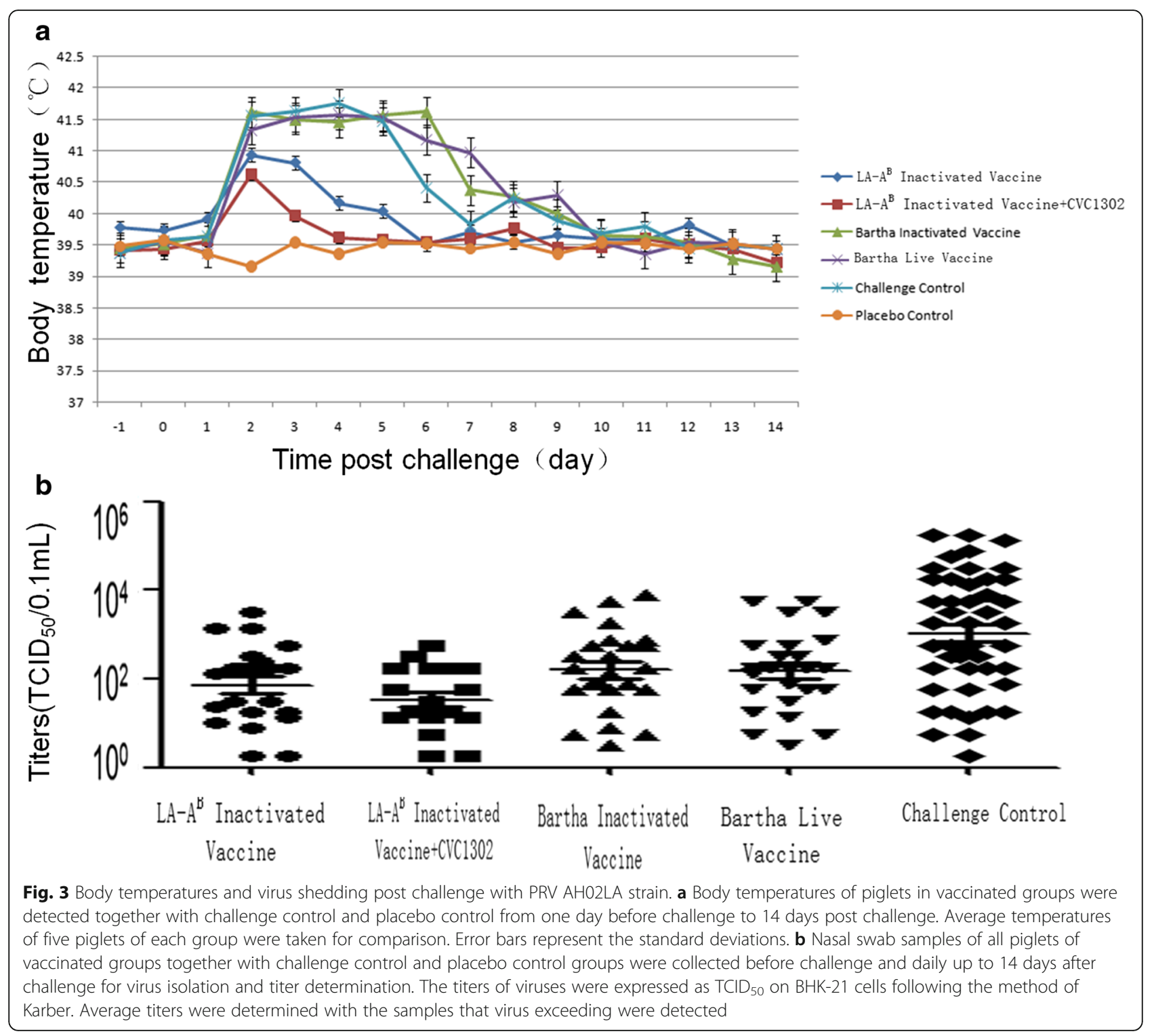

lower than through I.N. way. This difference is presumed to be associated with the increased titers of virus after replication on epithelial cells of respiratory tract. To mimick the nature infection of domestic pigs genuinely, we did the challenge protection test through I.N. way for evaluation of the vaccines in this study.

\section{Conclusion}

The infectious clone consists of the whole genome of PRV emerging variant AH02LA strain. Inactivated vaccine made of PRV LA- $\mathrm{A}^{\mathrm{B}}$ strain can provide better clinical protection than vaccines made of Bartha K61. In addition, significant decrease of virus shedding was observed post lethal challenge when $\mathrm{LA}-\mathrm{A}^{\mathrm{B}}$ inactivated vaccine were adjuvanted with CVC1302.

\section{Abbreviations}

BAC: Bacterial artificial chromosome; CEC: Chicken embryo cell; CPE: Cytopathogenic effects; E.coli: Escherichia coli; ELISA: Enzyme-linked immunosorbent assay; gB: glycoprotein B; gE: glycoprotein E; gl: glycoprotein I; Hpi: Hour post infection; I.M: Intramuscularly; I.N: Intranasally; LD 50 : 50\% lethal dose; MCMV: Mouse cytomegalovirus; MDP: Muramyl dipeptide; MOI: Multiplicity of infection; MPLA: Monophosphoryl lipid; NA: Neutralizing antibodies; ORF: Open reading frame; PC: Post challenge; PV: Post vaccination; PBS: Phosphate buffer saline; PCR: Polymerase chain reaction; PRV: Pseudorabies virus; RFLP: Restriction fragment length polymorphism; $\operatorname{TCID}_{50}: 50 \%$ tissue culture infectious dose; TK: Thymidine kinase; VN: Virus neutralization

\section{Acknowledgements}

We thanks professor Nikolaus Osterrieder at the Free University of Berlin for kindly providing E.coli GS1783. We thanks Dr Qisheng Zheng for kindly providing adjuvant CVC1302.

\section{Funding}

This study was supported by the Special Fund for Agro-Scientific Research in the Public Interest (201303046), Program for Independence and Innovation 
in Agricultural Sciences of Jiangsu Province (CX(12)3061 and CX(14)2084), and the Natural Science Foundation of Jiangsu Province (BK20131334).

\section{Availability of supporting data}

The data set(s) supporting the results of this article is included within the article. And all row data are available in National Research Center of Engineering and Technology for Veterinary Biologicals/Institute of Veterinary Medicine, Jiangsu Academy of Agricultural Sciences, Nanjing Jiangsu, China upon official request via mail (jcwang@263.net).

\section{Authors' contributions}

JW and JH designed the generation of BAC and related mutants. JW, YQ, YG and $\mathrm{CL}$ contributed to the design and performance of animal tests. $\mathrm{RG}, \mathrm{YG}$, $\mathrm{CL}, \mathrm{YL}$ and $\mathrm{MX}$ constructed the bacterial artificial chromosome of PRV and the related studies. JW ZW and RG constructed the gE deleted vaccine. ZW, $J W, Y Q$ and $R G$ investigated the related properties of the vaccine and performed the animal experiments and related tests. All authors approved the final manuscript.

\section{Competing interests}

The authors declare that they have no conflicts of interest.

\section{Consent for publication}

Not applicable.

\section{Ethics approval}

All animal tests were approved by the Institutional Animal Care and Use Committee and were conducted following the guidelines of the Institutional Biosafety Committee at the Jiangsu Academy of Agriculture Sciences. Experiments involving virulent PRV were conducted under Biosafety Level $2+$ containment. At the end of test, all survived piglets were euthanized by injection of pentobarbital.

\section{Author details}

'National Research Center of Engineering and Technology for Veterinary Biologicals/Institute of Veterinary Medicine, Jiangsu Academy of Agricultural Sciences, Nanjing, Jiangsu 210014, China. ${ }^{2}$ College of Veterinary Medicine, Nanjing Agricultural University, Nanjing 210095, China. ${ }^{3}$ Jiangsu Co-innovation Center for Prevention and Control of Important Animal Infectious Diseases and Zoonoses, Yangzhou, China.

Received: 21 June 2016 Accepted: 24 November 2016

Published online: 07 December 2016

\section{References}

1. Zimmerman JJ, Karriker LA, Ramirez A, Schwartz KJ, Stevenson GW. Diseases of Swine. 10th Edition. Ames: lowa State University Press, Wiley-Blackwell. 2014;1541-73.

2. Van Nes A, Stegeman JA, De Jong MC, Loeffen WL, Kimman TG JH. No massive spread of pseudorabies virus in vaccinated sow herds. Vet Microbiol. 1997;55(1-4):147-51.

3. Lyman M, Demmin G, Banfield B. The attenuated pseudorabies virus strain Bartha fails to package the tegument proteins Us3 and VP22. J Virol. 2003; 77(2):1403-14.

4. Wang C, Yuan J, Qin H, Luo Y, Cong X, Chen J, Li S, Sun Y, Qiu H. A novel $\mathrm{gE}$-deleted pseudorabies virus (PRV) provides rapid and complete protection from lethal challenge with the PRV variant emerging in BarthaK61-vaccinated swine population in China. Vaccine. 2014;32(27):3379-85.

5. Ye C, Zhang Q, Tian Z, Zheng H, Zhao K, Liu F, Guo J, Tong W, Jiang C, Wang $\mathrm{S}$, et al. Genomic characterization of emergent pseudorabies virus in China reveals marked sequence divergence: Evidence for the existence of two major genotypes. Virology. 2015;483:32-43.

6. Yu X, Zhou Z, Hu D, Zhang Q, Han T, Li X, Gu X, Yuan L, Zhang S, Wang B, et al. Pathogenic pseudorabies virus, China, 2012. Emerg Infect Dis. 2014;20(1):102-4.

7. Wang Y, Qiao S, Li X, Xie W, Guo J, Li Q, Liu X, Hou J, Xu Y, Wang L, et al. Molecular epidemiology of outbreak-associated pseudorabies virus (PRV) strains in central China. Virus Genes. 2015:50(3):401-9.

8. An T, Peng J, Tian Z, Zhao H, Li N, Liu Y, Chen J, Leng C, Sun Y, Chang D, et al. Pseudorabies virus variant in bartha-k61-vaccinated pigs, china, 2012 Emerg Infect Dis. 2013;19(11):1749-55.
9. Sun Y, Luo Y, Wang C, Yuan J, Li N, Song K, Qiu H. Control of swine pseudorabies in China: Opportunities and limitations. Vet Microbiol. 2016; 183:119-24.

10. Peng JM, An TQ, Zhao HY, Liu YM, Chen JZ, Leng CL, Sun Y, Chang D, Tian $Z$ J, Tong GZ. Identification and antigenic variation of new epidemiology of pseudorabies virus from swine. Chin J Prev Vet Med. 2013:01:1-4.

11. Serena M, Geisler C, Metz G, Mórtola E, Echeverría M. Production of pseudorabies virus recombinant glycoprotein $B$ and its use in an agar gel immunodiffusion (AGID) test for detection of antibodies with sensitivity and specificity equal to the virus neutralization assay. J Virol Methods. 2016:230:9-12

12. Yang Q, Sun Z, Tan F, Guo L, Wang Y, Wang J, Wang Z, Wang L, Li X, Xiao Y, et al. Pathogenicity of a currently circulating Chinese variant pseudorabies virus in pigs. World J Virol. 2016;5(1):23-30.

13. Xiang S, Zhou Z, Hu X, Li Y, Zhang C, Wang J, Li X, Tan F, Tian K. Complete Genome Sequence of a Variant Pseudorabies Virus Strain Isolated in Central China. Genome Announc. 2016:4(2). doi: 10.1128/genomeA.00149-16

14. Papageorgiou K, Suárez N, Wilkie G, Filioussis G, Papaioannou N, Nauwynck H, Davison A, SK K. Genome sequences of two pseudorabies virus strains isolated in Greece. Genome Announc. 2016;4(1). doi: 10.1128/genomeA.01624-15.

15. Zhang C, Guo L, Jia X, Wang T, Wang J, Sun Z, Wang L, Li X, Tan F. Construction of a triple gene-deleted Chinese Pseudorabies virus variant and its efficacy study as a vaccine candidate on suckling piglets. Vaccine. 2015;33(21):2432-7.

16. Gu Z, Dong J, Wang J, Hou C, Sun H, Yang W, Bai J, Jiang P. A novel inactivated $\mathrm{gE} / \mathrm{gl}$ deleted pseudorabies virus (PRV) vaccine completely protects pigs from an emerged variant PRV challenge. Virus Res. 2014; 195(14):57-63

17. Miller L, Bayles D, Zanella E, Lager K. Effects of Pseudorabies Virus Infection on the Tracheobronchial Lymph Node Transcriptome. Bioinform Biol Insights. 2016;9 Suppl 2:25-36.

18. Wu C, Liao C, Chi J, Chien M, Huang C. Growth properties and vaccine efficacy of recombinant pseudorabies virus defective in glycoprotein $E$ and thymidine kinase genes. J Biotechnol. 2016:229:58-64.

19. Morgan R, Cantello J, McDermott C. Transfection of chicken embryo fibroblasts with Marek's disease virus DNA. Avian Dis. 1990;34:345-51.

20. Boussif O, Lezoualc'h F, Zanta M, Mergny M, Scherman D, Demeneix B, Behr J. A versatile vector for gene and oligonucleotide transfer into cells in culture and in vivo: polyethylenimine. Proc Natl Acad Sci U S A. 1995;92:7297-301.

21. Muyrers J, Zhang Y, Testa G, Stewart A. Rapid modification of bacterial artificial chromosomes by ET-recombination. Nucleic Acids Res. 1999;27.

22. Narayanan $K$, Williamson $R$, Zhang $Y$, Stewart A, loannou P. Efficient and precise engineering of a $200 \mathrm{~kb}$ beta-globin human/bacterial artificial chromosome in E. coli DH10B using an inducible homologous recombination system. Gene Ther. 1999;6:442-7.

23. Tischer B, Smith G, Osterrieder N. En passant mutagenesis: a two step markerless red recombination system. Methods Mol Biol. 2010;634:421-30.

24. Schumacher D, Tischer B, Fuchs W, Osterrieder N. Reconstitution of Marek's disease virus serotype 1 (MDV-1) from DNA cloned as a bacterial artificial chromosome and characterization of a glycoprotein B-negative MDV-1 mutant. J Virol. 2000;74:11088-98.

25. Shizuya H, Birren B, Kim U, Mancino V, Slepak T, Tachiiri Y, Simon M. Cloning and stable maintenance of 300-kilobase-pair fragments of human DNA in Escherichia coli using an F-factor-based vector. Proc Natl Acad Sci U S A. 1992;89:8794-7.

26. Wang J, Osterrieder N. Generation of an infectious clone of duck enteritis virus and generation of a vectored DEV expressing hemagglutinin of $\mathrm{H} 5 \mathrm{~N} 1$ avian influenza virus. Virus Res. 2011;159(1):23-31.

27. Rudolph J, O'Callaghan D, Osterrieder N. Cloning of the genomes of equine herpesvirus type1 (EHV-1) strains KyA and racL11 as bacterial artificial chromosomes (BAC). J Vet Med B Infect Dis Vet Public Health. 2002:49:31-6.

28. Lerma L, Muñoz A, Wagner S, Dinu M, Martín B, Tabarés E. Construction of recombinant pseudorabies viruses by using PRV BACs deficient in IE180 or pac sequences: Application of $\mathrm{VBAC} 90 \mathrm{D}$ recombinant virus to production of PRV amplicons. Virus Res. 2016;213:274-82.

29. Fan J, Zeng X, Zhang G, Wu Q, Niu J, Sun B, Xie Q, Ma J. Molecular characterization and phylogenetic analysis of pseudorabies virus variants isolated from Guangdong Province of Southern China during 2013-2014. J Vet Sci. 2015; [Epub ahead of print].

30. Le Luduec J, Debeer S, Piras F, Andréoni C, Boudet F, Laurent P, Kaiserlian D, Dubois $B$. Intradermal vaccination with un-adjuvanted sub-unit vaccines 
triggers skin innate immunity and confers protective respiratory immunity in domestic swine. Vaccine. 2016;34(7):914-22.

31. Liang X, Sun L, Yu T, Pan Y, Wang D, Hu X, Fu Z, He Q, Cao G. A CRISPR/ Cas9 and Cre/Lox system-based express vaccine development strategy against re-emerging Pseudorabies virus. Sci Rep. 2016;6:19176.

32. Messerle M, Crnkovic I, Hammerschmidt W, Ziegler H, Koszinowski U. Cloning and mutagenesis of a herpesvirus genome as an infectious bacterial artificial chromosome. Proc Natl Acad Sci U S A. 1997;94:14759-63.

33. Osterrieder N, Schumacher D, Trapp S, Beer M, von Einem J, Tischer K. Establishment and use of infectious bacterial artificial chromosome (BAC) DNA clones of animal herpesviruses. Berl Munch Tierarztl Wochenschr. 2003; 116:373-80.

34. Rosas C, Van de Walle G, Metzger S, Hoelzer K, Dubovi E, Kim S, Parrish C, Osterrieder N. Evaluation of a vectored equine herpesvirus type 1 (EHV-1) vaccine expressing $\mathrm{H} 3$ haemagglutinin in the protection of dogs against canine influenza. Vaccine. 2008;26:2335-43.

35. Wang J, Ge A, Xu M, Wang Z, Qiao Y, Gu Y, Liu C, Liu Y, Hou J. Construction of a recombinant duck enteritis virus (DEV) expressing hemagglutinin of $\mathrm{H} 5 \mathrm{~N} 1$ avian influenza virus based on an infectious clone of DEV vaccine strain and evaluation of its efficacy in ducks and chickens. Virol J. 2015;12:126.

36. Yoon H, Eo S, Aleyas A, Cha S, Lee J, Chae J, Jang H, Cho E, Song H. Investigation of pseudorabies virus latency in nervous tissues of seropositive pigs exposed to field strain. J Vet Med Sci. 2006;68(2):143-8.

37. Wang D, Huang R. Effect of eradication of pseudorabies virus in breeding pig farms. Anhui Agri Sci Bull. 2009;15(24):30-1.

38. Li G. Control and eradication of pseudorabies in large-scale pig farms. Hubei J Anim Vet Sci. 2009;11:39-40.

\section{Submit your next manuscript to BioMed Central and we will help you at every step:}

- We accept pre-submission inquiries

- Our selector tool helps you to find the most relevant journal

- We provide round the clock customer support

- Convenient online submission

- Thorough peer review

- Inclusion in PubMed and all major indexing services

- Maximum visibility for your research

Submit your manuscript at www.biomedcentral.com/submit

C) Biomed Central 